\title{
WHEN DO THE SYMMETRIC TENSORS OF A COMMUTATIVE ALGEBRA FORM A FROBENIUS ALGEBRA?
}

\author{
ANNETTA G. ARAMOVA AND LUCHEZAR L. AVRAMOV
}

\begin{abstract}
For a commutative $k$-algebra $B$, consider the subalgebra $\left(B^{\otimes n}\right)^{S_{n}}$ of the $n$th tensor power of $B$, formed by the tensors invariant under arbitrary permutations of the indices. Necessary and sufficient conditions are found for $\left(B^{\otimes n}\right)^{S_{n}}$ to be Frobenius. When $\operatorname{dim}_{k} B \neq 2$, these say that $B$ is Frobenius and $n$ ! is invertible in $k$, unless $B$ is separable. Some additional cases occur for two-dimensional algebras in positive characteristic, depending on the divisibility of $n+1$.
\end{abstract}

1. Introduction. Let $k$ be a field, and let $B$ be a commutative $k$-algebra. In [1] G. Azumaya considers the following question, raised by $N$. Jacobson: If $B$ is Frobenius and $G$ is the group of order two generated by $\tau$, acting on $A=B \otimes B$ by $\tau\left(b_{1} \otimes b_{2}\right)=b_{2} \otimes b_{1}$, is the fixed subalgebra $A^{G}$ also Frobenius? (Recall that by definition, $B$ is Frobenius if it is finite-dimensional over $k$, and $B \simeq \operatorname{Hom}_{k}(B, k)$ as $B$-modules.) The main result of [1] shows that when $B$ is generated over $k$ by a single element, then $A^{G}$ is Frobenius if and only if the characteristic of $k$ is different from 2 , or $B$ is a direct product of separable field extensions. As an immediate corollary to our theorem below, we obtain a complete answer to Jacobson's question, by showing that the restriction on the number of generators is superfluous.

More generally, in this note we are concerned with the subalgebra of the $n$-fold $k$-tensor product $B^{\otimes n}$, consisting of symmetric tensors, i.e. the fixed subalgebra of $B^{\otimes n}$ under the action of the symmetric group $S_{n}$ :

$$
\tau\left(b_{1} \otimes \cdots \otimes b_{n}\right)=b_{\tau^{-1}(1)} \otimes \cdots \otimes b_{\tau^{-1}(n)}
$$

for $\tau \in S_{n}$. Our result gives a complete description of those $B$ and $n$, for which $\left(B^{\otimes n}\right)^{S_{n}}$ is Frobenius. In what follows, all algebras, unless expressly stated, are over $k$, and have a unit which is preserved by algebra homomorphisms; all unadorned tensor products are taken over $k$.

THEOREM. For a commutative algebra $B$, over a field $k$ of characteristic $p$, the symmetric tensors $\left(B^{\otimes n}\right)^{S_{n}}$ form a Frobenius algebra if and only if

(a) $B$ is Frobenius, and

(b) one of the following (disjoint) conditions holds:

(i) $p=0$;

(ii) $p>n$;

Received by the editors September 10, 1981.

1980 Mathematics Subject Classification. Primary 16A36, 13H10, 16A74, 13 B04.

Key words and phrases. Frobenius algebra, zero-dimensional Gorenstein ring, symmetric tensors, invariants of a finite group of automorphisms.

(C) 1982 American Mathematical Society $0002-9939 / 81 / 0000-0670 / \$ 02.50$ 
(iii) $0<p \leq n, B \simeq k[X] /\left(X^{2}\right)$, and $n=p^{j} b-1$ for some integers $j \geq 1$ and $1 \leq b \leq p-1$

(iv) $p=2, B$ is a quadratic purely inseparable field extension of $k$, and $n=2^{j}-1$ for some integer $j \geq 2$;

(v) $0<p \leq n$, and $B$ is the direct product of (a finite number of finite) separable field extensions.

REMARK. That condition (a) is necessary for $\left(B^{\otimes n}\right)^{S_{n}}$ to be Frobenius is almost immediate, and that (a) together with one of (i)-(v) is sufficient for this to hold, is rather easily seen. Hence, the bulk of our work below goes into showing that when $B$ and $\left(B^{\otimes n}\right)^{S_{n}}$ are both Frobenius, one of the conditions from (i)-(v) must be satisfied. However, for reasons of exposition, we have chosen a somewhat different arrangement of the material below.

REMARK. In commutative algebra, one usually speaks, meaning the same thing, of Gorenstein algebras, finite-dimensional over $k$, rather than of Frobenius ones. Hence a natural extension of the question raised in the title would be: When do the symmetric tensors of a finitely-generated commutative $k$-algebra form a Gorenstein ring?

That a rather different answer should be expected is indicated by the following immediate corollary of Watanabe's main result in [2.I]]: Suppose $p=0$, or $p>$ $n$, and let $B=k\left[X_{1}, \ldots, X_{m}\right]$ be the polynomial ring in $m$ indeterminates; then $\left(B^{\otimes n}\right)^{S_{n}}$ is Gorenstein if and only if either $m=1$, or $m$ is even.

\section{Preliminaries.}

(1) LEMMA. The k-vector spaces $B$ and $\left(B^{\otimes n}\right)^{S_{n}}$ are finite-dimensional or not alike. Moreover, if $e_{1}, \ldots, e_{m}$ is a $k$-basis of $B$, then a $k$-basis of $\left(B^{\otimes n}\right)^{S_{n}}$ is given by the elements

$$
\mathbf{o}_{n}\left[j_{1}, j_{2}, \ldots, j_{m}\right]=\sum a_{\tau^{-1}(1)} \otimes \cdots \otimes a_{\tau^{-1}(n)}
$$

where the summation is taken over a set of representatives of the left cosets of $S_{n} / S_{j_{1}} \times \cdots \times S_{j_{m}}$, with $j_{1}+\cdots+j_{m}=n$, and $a_{j}=e_{h}$ for $j_{1}+\cdots+j_{h-1}<j \leq$ $j_{1}+\cdots+j_{h}$, for $1 \leq h \leq m$. In particular, if $\operatorname{dim}_{k} B=m$, then $\operatorname{dim}_{k}\left(B^{\otimes n}\right)^{S_{n}}=$ $\left(\begin{array}{c}m+n-1 \\ n\end{array}\right)$.

PROOF. Left to the reader.

(2) LEMMA. Let $B=B_{1} \times \cdots \times B_{t}$ be a decomposition as a direct product of $k$-algebras. Then as $k$-algebras

$$
\left(B^{\otimes n}\right)^{S_{n}} \simeq \prod_{\substack{r_{1}+\ldots+r_{t}=n \\ r_{i} \geq 0}}\left(B_{1}^{\otimes r_{1}}\right)^{S_{r_{1}}} \otimes \cdots \otimes\left(B_{t}^{\otimes r_{t}}\right)^{S_{r_{t}}}
$$

Proof. Consider the $k$-linear map $f$ from the right side to the left one, obtained by composing the inclusion of each factor into the corresponding $\left(B_{1}^{\otimes r_{1}}\right) \otimes$ $\cdots \otimes\left(B_{t}^{\otimes r_{t}}\right)=B_{r_{1}, \ldots, r_{t}}$, with the map sending $b_{1} \otimes \cdots \otimes b_{n} \in B_{r_{1}, \ldots, r_{t}}$ to $\sum b_{\tau^{-1}(1)} \otimes \cdots \otimes b_{\tau^{-1}(n)}$, the summation being taken over a set of representatives of the left cosets of $S_{n} / S_{r_{1}} \times \cdots \times S_{r_{t}}$. Clearly, $f$ is injective. To see that it is an isomorphism, suppose first that $\operatorname{dim} B_{i}=m_{i}<\infty$ for $1 \leq i \leq t$. By (1) the dimension of the left side is $\left(\begin{array}{c}m+n-1 \\ n\end{array}\right)$, while that of the right one 
is $\sum_{r_{1}+\cdots+r_{t}=n}\left(\begin{array}{c}m_{1}+r_{1}-1 \\ r_{1}\end{array}\right) \ldots\left(\begin{array}{c}m_{t}+r_{t}-1 \\ r_{t}\end{array}\right)$. These integers coincide-compare the coefficient of $X^{n}$ in the identity of formal power series

$$
(1-X)^{-m}=\prod_{i=1}^{t}(1-X)^{-m_{i}},
$$

hence $f$ is bijective. To treat the general case, note that if $B^{\prime}=B_{1}^{\prime} \times \cdots \times B_{t}^{\prime}\left(B_{i}^{\prime} \subset\right.$ $\left.B_{i}\right)$ is a finite-dimensional subspace of $B$, then $f$ maps $\prod_{r_{1}+\cdots+r_{t}=n}\left(B_{1}^{\prime \otimes r_{1}}\right)^{S_{r_{1}}} \otimes$ $\cdots \otimes\left(B_{t}^{\prime \otimes r_{t}}\right)^{S_{r_{t}}}$ into $\left(B^{\prime \otimes n}\right)^{S_{n}}$, and this is an isomorphism by the case already settled. Since $f$ commutes with direct limits, it is an isomorphism for every $B$.

It remains to observe that $f$ is a $k$-algebra map.

(3) COROLLARY. $\left(B^{\otimes n}\right)^{S_{n}}$ is Frobenius if and only if $\left(B_{i}^{\otimes r_{i}}\right)^{S_{r_{i}}}$ is Frobenius for $1 \leq i \leq t$ and $r_{1}+\cdots+r_{t}=n$.

PROOF. It is clear that a direct product of algebras is Frobenius if and only if each factor is such, and the corresponding property holds, in the commutative case, also for the tensor product: $\mathrm{cf}$. [3, Theorem 1'].

3. The local case. Recall that a finite-dimensional commutative local $k$-algebra $(B, \mathbf{m})$ is Frobenius if and only if $\operatorname{dim}_{B / \mathbf{m}}(0: \mathbf{m})=1$.

(4) LemMA. Let $0<p \leq n$, and $B=k[X] /\left(X^{2}\right)$. Then $\left(B^{\otimes n}\right)^{S_{n}}$ is Frobenius if and only if $n=p^{j} b-1$, for some integers $j \geq 1,1 \leq b \leq p-1$.

PROOF. Identifying $B^{\otimes n}$ with the graded $k$-algebra

$$
k\left[X_{1}, \ldots, X_{n}\right] /\left(X_{1}^{2}, \ldots, X_{n}^{2}\right),
$$

(1) shows that the elementary symmetric functions $\sigma_{q}(1 \leq q \leq n)$, together with 1 , provide a homogeneous basis for the symmetric tensors. The product rule being $\sigma_{r} \sigma_{q}=\left(\begin{array}{c}r+q \\ q\end{array}\right) \sigma_{r+q}$, where $\sigma_{i}=0$ for $i>n$, one sees, using the formula

$$
\left(\begin{array}{l}
a \\
b
\end{array}\right) \equiv \prod\left(\begin{array}{l}
a_{i} \\
b_{i}
\end{array}\right) \bmod (p)
$$

(for $a=\sum a_{i} p^{i}, b=\sum b_{i} p^{i}, 0 \leq a_{i} \leq p-1,0 \leq b_{i} \leq p-1$ ) that $\sigma_{i}^{p}=0$ for all $i$, and that if $q$ is not a power of $p$, then $\sigma_{q}$ is expressible as a product of elements of lower degree. In particular, the elements $\sigma_{p^{i}}$, when $1 \leq p^{i} \leq n$, generate $\left(B^{\otimes n}\right)^{S_{n}}$ as a $k$-algebra.

Let now $j$ and $b$ be the integers uniquely defined by the inequalities $b p^{j}-1 \leq$ $n<(b+1) p^{j}-1,(p \leq n), 1 \leq b \leq p-1$. The element $s=\sigma_{1}^{p-1} \cdots \sigma_{p^{j-1}}^{p-1} \sigma_{p^{j}}^{b-1}$ of degree $b p^{j}-1$ is in the socle of $\left(B^{\otimes n}\right)^{S_{n}}: s \sigma_{p^{i}}$ is divisible by $\sigma_{p^{i}}^{p}=0$ for $0 \leq$ $i \leq j-1$, while $\operatorname{deg}\left(s \sigma_{p^{j}}\right)=(b+1) p^{j}-1>n$. Hence, in case $n>b p^{j}-1$, the symmetric tensors are not a Frobenius algebra, since a nonzero element of degree $n$ gives a linearly independent from $s$ element of the socle. On the other hand, assume $n=b p^{j}-1$; then $\sigma_{p^{j}}^{b}=0$, since its degree is $b p^{j}>n$. Hence every nonzero product of generators has the form $x=\sigma_{1}^{a_{0}} \cdots \sigma_{p^{j-1}}^{a_{j-1}} \sigma_{p^{j}}^{a_{j}}$, with $0 \leq a_{i} \leq$ $p-1(0 \leq i \leq j-1), 0 \leq a_{j} \leq b-1$, and these form a $k$-basis of $\left(B^{\otimes n}\right)^{S_{n}}$. Taking $y=\sigma_{1}^{c_{0}} \cdots \sigma_{p^{j}}^{c_{j}}$ with $\bar{c}_{i}=p-1-a_{i}(0 \leq i \leq j-1), c_{j}=b-1-a_{j}$, we see that $x \cdot y=s$, hence $s$ generates the socle of $\left(B^{\otimes n}\right)^{S_{n}}$, and we are done. 
(5) LEMMA. Let $(B, \mathbf{m})$ be a local commutative Frobenius $k$-algebra, such that $B / \mathbf{m} \simeq k$, and $\operatorname{dim}_{k} B \geq 3$. Then $\left(B^{\otimes n}\right)^{S_{n}}$ is Frobenius if and only if either $p=0$, or $p>n$.

Proof. Choose a generator $s$ of $(0: \mathrm{m})$. Since $B^{\otimes n}$ is a local Frobenius $k$ algebra, with socle generated by $s \otimes \cdots \otimes s$ ( $n$ factors), and this element is fixed by all $\tau \in S_{n}$, the invariant subring is (local) Frobenius if either $p=0$, or $p>n$ by an easy lemma of Watanabe [2.I, Lemma 4]. Hence, from now on, we suppose $0<p \leq n$, and fix a basis $e_{1}, \ldots, e_{m}$ of $B$ such that $e_{1}=1, e_{m}=s, e_{m-1}=t$, where $t \in\left(0: \mathbf{m}^{2}\right) \backslash(0: \mathbf{m})$ (this is not empty since $B$ is not a field). Note that $t e_{i}=\alpha_{i} s$ for some $\alpha_{i} \in k(2 \leq i \leq m)$. Our purpose is to produce a symmetric tensor $s^{\prime} \neq 0$, not proportional to $s \otimes \cdots \otimes s$, which annihilates every basis element $x=\mathbf{o}_{n}\left[j_{1}, \ldots, j_{m}\right]$ with $j_{1}<n$. There are several cases to be considered.

Case I. $n+1=p q+r$, with $1 \leq r \leq p-1$.

Set

$$
s^{\prime}=\mathbf{o}_{n}[0, \ldots, 0, r, n-r]
$$

and note that $s^{\prime} x=0$ if $j=j_{2}+\cdots+j_{m}>r$. If $j \leq r$, then $s^{\prime} x$ is a sum of decomposable tensors with $r-j$ entries equal to $t$, and $n-r+j$ entries equal to $s$, each summand appearing with a coefficient $\alpha_{2}^{j_{2}} \alpha_{3}^{j_{3}} \cdots \alpha_{m}^{j_{m}}$. Since all these tensors are in the same $S_{n}$-orbit, we have by (1)

$$
s^{\prime} x=A_{j_{2}, \ldots, j_{m}} \alpha_{2}^{j_{2}} \cdots \alpha_{m}^{j_{m}} \mathbf{o}_{n}[0, \ldots, 0, r-j, n-r+j]
$$

for some integer $A_{j_{2}}, \ldots, j_{m}$, which is equal to the number of times the element

$$
w=\mathbf{o}_{r-j}[0, \ldots, 0, r-j, 0] \otimes \mathbf{o}_{n-r+j}[0, \ldots, 0, n-r+j]
$$

occurs in $s^{\prime} x$. Writing

$$
\begin{aligned}
s^{\prime} & =\mathbf{o}_{r-j}[0, \ldots, 0, r-j, 0] \otimes \mathbf{o}_{n-r+j}[0, \ldots, 0, j, n-r]+z, \\
x & =\mathbf{o}_{r-j}[r-j, 0, \ldots, 0] \otimes \mathbf{o}_{n-r+j}\left[n-r, j_{2}, \ldots, j_{m}\right]+y,
\end{aligned}
$$

note that $w$ appears only from products of elements not in $z$ with elements not in $y$, and this happens exactly once for each summand in $x-y$. Hence

$$
\begin{aligned}
A_{j_{2}, \ldots, j_{m}} & =(n-r+j) ! /\left((n-r) ! j_{2} ! \cdots j_{m} !\right) \\
& =(n-r+j) \cdots(n-r+1) /\left(j_{2} ! \cdots j_{m} !\right)
\end{aligned}
$$

and this integer is divisible by $p$, since the numerator contains a factor $n-r+1=$ $p q$, while the denominator is prime to $p$ (because $j_{i} \leq j \leq r<p$ for $2 \leq i \leq m$ ).

Case IIa. $n+1=p q$, and $\operatorname{dim}_{k}\left(0: \mathbf{m}^{2}\right)>2$.

Choose a second element $u \in\left(0: \mathrm{m}^{2}\right)$ such that $s, t, u$ are linearly independent, and choose a basis of $B$ for which $e_{m-2}=u$, in addition to the previous conditions. Let $u e_{i}=\beta_{i} s\left(\beta_{i} \in k, 2 \leq i \leq m\right)$, and set

$$
s^{\prime}=\mathbf{o}_{n}[0, \ldots, 0,1, p-1, n-p] \text {. }
$$

Then $s^{\prime} x=0$ when $j=j_{2}+\cdots+j_{m}>p$. Otherwise, this product is expressed as a linear combination of decomposable tensors having $n-p+j$ entries equal to $s$, at most one entry equal to $u$, and the remaining ones equal to $t$; also, note that an entry equal to $u$ will appear only in case $j \leq p-1$. From (1) we deduce that

$$
\begin{aligned}
s^{\prime} x= & B_{j_{2}, \ldots, j_{m}} \alpha_{2}^{j_{2}} \cdots \alpha_{m}^{j_{m}} \mathbf{o}_{n}[0, \ldots, 0,1, p-j-1, n-p+j] \\
& +\sum_{\substack{h=2 \\
j_{h} \geq 1}}^{m} C_{j_{2}, \ldots, j_{m}}^{h} \beta_{h} \alpha_{2}^{j_{2}} \cdots \alpha_{h}^{j_{h}-1} \cdots \alpha_{m}^{j_{m}} \mathbf{o}_{n}[0, \ldots, 0, p-j, n-p+j]
\end{aligned}
$$


where $B_{j_{2}, \ldots, j_{m}}$ and $C_{j_{2}, \ldots, j_{m}}^{h}$ are integers, the first of which is zero when $j=p$. Moreover, $B_{j_{2}, \ldots, j_{m}}$ is the number of times the summand

$$
u \otimes o_{p-j-1}[0, \ldots, 0, p-j-1,0] \otimes o_{n-p+j}[0, \ldots, 0, n-p+j]
$$

appears in $s^{\prime} x$, and similarly $C_{j_{2}, \ldots, j_{m}}^{h}$ is the number of times the summand

$$
\mathbf{o}_{p-j}[0, \ldots, 0, p-j, 0] \otimes \mathbf{o}_{n-p+j}[0, \ldots, 0, n-p+j]
$$

comes up with a coefficient $\beta_{h} \alpha_{2}^{j_{2}} \cdots \alpha_{h}^{j_{h}-1} \cdots \alpha_{m}^{j_{m}}$. Writing

$$
\begin{aligned}
& s^{\prime}=u \otimes o_{p-j-1}[0, \ldots, 0, p-j-1,0] \otimes o_{n-p+j}[0, \ldots, 0, j, n-p]+z, \\
& x=1 \otimes o_{p-j-1}[p-j-1,0, \ldots, 0] \otimes o_{n-p+j}\left[n-p, j_{2}, \ldots, j_{m}\right]+y,
\end{aligned}
$$

we conclude as in Case I that

$$
B_{j_{2}, \ldots, j_{m}}=(n-p+j) \cdots(n-p+1) /\left(j_{2} ! \cdots j_{m} !\right),
$$

which is divisible by $p$. On the other hand, from

$$
\begin{aligned}
s^{\prime} & =\mathbf{o}_{p-j}[0, \ldots, 0, p-j, 0] \otimes \mathbf{o}_{n-p+j}[0, \ldots, 0,1, j-1, n-p]+z^{\prime}, \\
x & =\mathbf{o}_{p-j}[p-j, 0, \ldots, 0] \otimes \mathbf{o}_{n-p+j}\left[n-p, j_{2}, \ldots, j_{m}\right]+y^{\prime}
\end{aligned}
$$

we see that

$$
C_{j_{2}, \ldots, j_{m}}^{h}=(n-p+j) \cdots(n-p+1) /\left(j_{2} ! \cdots\left(j_{h}-1\right) ! \cdots j_{m} !\right),
$$

and this integer also is divisible by $p$.

Case IIb. $n+1=p q$, and $\operatorname{dim}_{k}\left(0: \mathbf{m}^{2}\right)=2$.

Since $\left(0: \mathbf{m}^{2}\right) /(0: \mathbf{m}) \simeq\left(\mathbf{m} / \mathbf{m}^{2}\right)^{*}$ from the Frobenius condition, one necessarily has $B \simeq k[X] /\left(X^{m}\right), m \geq 3$. In the basis $e_{i}=X^{i-1}+\left(X^{m}\right), 1 \leq i \leq m$, set

$$
s^{\prime}=\mathbf{o}_{n}[0, \ldots, 0,1, p-1, n-p] \text {. }
$$

Clearly, $s^{\prime}$ annihilates $x$ if either $j_{h}>0$ for some $h \geq 4$, or $j_{3}>1$, or $j=$ $j_{2}+j_{3}>p$. In case $j_{h}=0$ for $h \geq 3$, and $j=j_{2} \leq p$, one has

$s^{\prime} x=D \mathbf{o}_{n}[0, \ldots, 0,1, p-j-1, n-p+j]+E \mathbf{o}_{n}[0, \ldots, 0, p-j+1, n-p+j-1]$

where $D=0$ if $j=p$. Arguing as above, one finds $D=\left(\begin{array}{c}n-p+j \\ j\end{array}\right)$ (when $j<p$ ), and $E=(p-j+1)\left(\begin{array}{c}n-p+j-1 \\ j-1\end{array}\right)$; both are divisible by $p$. There is just one more possibility, namely $x=\mathbf{o}_{n}[n-j, j-1,1,0, \ldots, 0], j \leq p$. Here

$$
s^{\prime} x=F \mathbf{o}_{n}[0, \ldots, 0, p-j, n-p+j]
$$

and one obtains for $F$ the value $j \cdot\left(\begin{array}{c}n-p+j \\ j\end{array}\right)$, which is a multiple of $p$.

The proof of the lemma is now complete.

4. Proof of the theorem. It is known that the $k$-algebra $B$ is Frobenius if and only if the $\bar{k}$-algebra $B \otimes_{k} \bar{k}$ is Frobenius, $\bar{k}$ being the algebraic closure of $k$. Moreover, the $n$-fold tensor product $C$ of $B \otimes_{k} \bar{k}$ with itself over $\bar{k}$ is isomorphic to $\left(B^{\otimes n}\right) \bigotimes_{k} \bar{k}$, this isomorphism commutes with the $S_{n}$-actions on $C$ and on $\left(B^{\otimes n}\right) \otimes_{k} \bar{k}$, and $C^{S_{n}} \simeq\left(B^{\otimes n}\right)^{S_{n}} \otimes_{k} \bar{k}$. Hence, we can assume $k$ is algebraically closed.

Suppose $\left(B^{\otimes n}\right)^{S_{n}}$ is Frobenius. Then $\operatorname{dim}_{k} B$ is finite by (1), hence $B$ has a decomposition $B=B_{1} \times \cdots \times B_{t}$ into finite-dimensional local $k$-algebras with residue field $k$. Supposing $B$ is not Frobenius, there must be a $B_{i}$ with two linearly independent elements $s_{1}, s_{2}$ in its socle. But then $s_{1} \otimes \cdots \otimes s_{1}$ and $s_{2} \otimes \cdots \otimes s_{2}$ 
( $n$ factors each) are linearly independent elements in the socle of $\left(B_{i}^{\otimes n}\right)^{S_{n}}$, hence this algebra is not Frobenius. By (3) this is a contradiction to the hypothesis on $\left(B^{\otimes n}\right)^{S_{n}}$. Thus, what remains to be done is, assuming $B$ Frobenius, to show that $\left(B^{\otimes n}\right)^{S_{n}}$ is Frobenius if and only if one of the conditions from (i)-(v) (in (b) of the theorem) holds.

By (3), $\left(B^{\otimes n}\right)^{S_{n}}$ is Frobenius if and only if this is true for $\left(B_{i}^{\otimes r_{i}}\right)^{S_{r_{i}}}$ with $1 \leq$ $i \leq t$ and $r_{1}+\cdots+r_{t}=n$. Applying (5), we see that when $\operatorname{dim}_{k} B_{i} \geq 3$ for some $i$, this is fulfilled if and only if either $p=0$, or $p>n$.

In what follows, we assume $0<p \leq n$, and $\operatorname{dim}_{k} B_{i} \leq 2$ for $1 \leq i \leq t$. When $\operatorname{dim}_{k} B_{i}=2$ for some $i, t \geq 2$ is impossible, since by (3) and (4) one must have $n=p^{j} b-1$ and $n-1=p^{h} c-1$ with $j, h \geq 1$ and $1 \leq b, c \leq p-1$. Hence in this case $B \simeq k[X] /\left(X^{2}\right)$, and $\left(B^{\otimes n}\right)^{S_{n}}$ is Frobenius if and only if $n=p^{j} b-1$. But all algebras, which after extension of scalars to the algebraic closure, become isomorphic to $k[X] /\left(X^{2}\right)$, are described by conditions (iii) and (iv) of the theorem.

Finally, condition ( $v$ ) of the theorem ( $k$ arbitrary), becomes equivalent, after passing to the algebraic closure, to the equalities $\operatorname{dim}_{k} B_{i}=1(1 \leq i \leq t)$, and in this case

$$
\left(B^{\otimes n}\right)^{S_{n}} \simeq k^{\left({ }^{n+t-1}\right)}
$$

is clearly Frobenius.

\section{REFERENCES}

1. G. Azumaya, Fixed subalgebra of a commutative Frobenius algebra, Proc. Amer. Math. Soc. 81 (1981), 213-216.

2. K. Watanabe, Certain invariant subrings are Gorenstein. I and II, Osaka J. Math. 11 (1974), 1-8, 379-388.

3. K. Watanabe, T. Ishikawa, S. Tachibana and K. Otsuka, On tensor products of Gorenstein rings, J. Math. Kyoto Univ. 9 (1969), 413-423.

institute of Mathematics, Bulgarian Academy of Sciences, ul. "Akad. G. BONCEV" 8, 1113 SOFIA, BULGARIA 